\title{
Impactos socioeconômicos da expansão do setor sucroenergético no Estado de São Paulo, entre 2005 e 2009
}

\author{
Palavras-chave \\ desenvolvimento, \\ setor sucroenergético, \\ dados em painel

\section{Classificação JEL} \\ O13, Q16, C23

\section{Keywords} \\ development, sugar and \\ ethanol sector, panel data
}

\section{JELClassification}

O13, Q16, C23

\section{Resumo}

O objetivo deste estudo é identificar os impactos socioeconômicos do crescimento do setor sucroenergético no Estado de São Paulo. Para tanto, foram utilizados dados em painel abrangendo todos os municípios, entre os anos de 2005 a 2009, sendo o modelo ajustado com procedimentos alternativos dessa metodologia. Os citados impactos foram avaliados considerando os aspectos de emprego/renda, educação e saúde. Foram analisados também os impactos do crescimento do setor sucroenergético sobre essas variáveis tomadas de forma agregada, para o índice consolidado de desenvolvimento. Os resultados do estudo permitem concluir que existe interação positiva e significativa entre a expansão do setor sucroenergéticono Estado de São Paulo e o emprego/renda. Todavia, não foram observadas evidências acerca do efeito expressivo da expansão do setor sobre a educação e a saúde.

\author{
Mirian Rumenos Piedade Bacchi* \\ Carlos Eduardo Caldarelli**
}

\section{Abstract}

This paper aims to identify the socialeconomic impacts of the expansion of the sugar and ethanol sector in the Sao Paulo state. Panel data were used for all municipalities from 2005 to 2009 making some adjustments to the model using different techniques of this methodology. The impacts were evaluated considering information about employment/income, education and health. Additionally, the impacts on those variables on an aggregate basis, measured by the index of development were also analyzed. The results of this research indicate that there is a significant and positive interaction between the sugarcane supply chain production and employment / income (positive elasticity) in the Sao Paulo state. However, the study does not show evidence that there is a significant interaction between the sector expansion and the levels of education and health. 


\section{1_Introdução}

A expansão do setor sucroenergético brasileiro faz com que surjam questionamentos ligados a aspectos ambientais, ao uso da terra e ao bem-estar da sociedade da área de abrangência, sendo este último assunto o objeto de análise do presente estudo.

O Brasil é o maior produtor de cana-de-açúcar do mundo, matéria-prima que permite a fabricação do açúcar, importante alimento energético na dieta da população brasileira - especialmente daqueles que têm baixo poder aquisitivo -, e do etanol, que é o combustível de maior expressão na atualidade entre os renováveis. $\mathrm{O}$ etanol é menos poluente que os combustíveis fósseis e também que os renováveis elaborados com outras matérias-primas que não a cana-de-açúcar (Bacchi, 2007).

O setor sucroenergético brasileiro, e mais especificamente o de biocombustível derivado da cana-de-açúcar, tem recebido atenção especial por parte dos países que buscam mitigar a emissão de gases poluentes introduzindo o etanol na sua matriz energética, sendo referência em fóruns internacionais que tratam de sustentabilidade. Nesse contexto, 0 Brasil pode ser um grande player no comércio internacional de etanol;embora esse mercado não seja grande nos dias de hoje, o seu potencial de crescimento é elevado.

A crescente frota brasileira de automóveis e comerciais leves, com grande representatividade dos bicombustíveis, impulsionada pelo aumento da renda da população e por isenções tributárias na comercialização desses veículos, associado ao fato de se ter preços internacionais e internos favoráveis em passado recente, tem feito com que a produção de cana-de-açúcar crescesse de forma significativa no país. No que diz respeito ao uso do etanol, além do seu uso como combustível de carros, comerciais leves e em motocicletas, passou esse combustível a ter maior expressão entre as matérias-primas de várias indústrias do setor químico (Satolo; Bacchi, 2012).
O Estado de São Paulo, de acordo com dados do SIDRA/ IBGE (2013), é o principal produtor brasileiro de cana-de-açúcar, respondendo, em2011, por aproximadamente 54,2\% da área plantada do país e por $58,2 \%$ da produção nacional de cana. São Paulo é também o maior produtor de açúcar e etanol do território nacional. Em 2000, os canaviais paulistas ocupavam uma área de quase 2,5 milhões de hectares, tendo passado para próximo a 5,2 milhões de hectares em 2011. Entre 2000 e 2011, a produção de cana aumentou em 237,53 milhões de toneladas, e a representatividade da atividade canavieira na área total plantada passou de 52,69\% para $74,68 \%$.

Segundo Nassar et al. (2008), no período de 2005 a 2008, houve um crescimento no Estado de mais de 1,8 milhão de hectares de área plantada com cana-de-açúcar, dos quais $53 \%$ ocorreram em substituição a áreas de pastagens e 46,7\% em substituição a outras atividades agrícolas.Dados do Canasat (2012) apontam que, das tradicionais regiões produtoras, ou seja, Ribeirão Preto, Araraquara, Piracicaba, Jaú e o Vale do Paranapanema, as lavouras canavieiras expandiram-separa outras regiões do Estado, especialmente para o norte e o oeste, na última década (2000/2010).

A grande relevância da atividade canavieira no contexto do agronegócio paulista e nacional justifica a análise de externalidades a ela relacionadas, podendo auxiliar no processo de definição de políticas públicas e setoriais.

Pretende-se, neste estudo,identificar os impactos socioeconômicos da expansão do setor sucroenergético no Estado de São Paulo, de 2005 a 2009, período em que ocorreu crescimento acentuado da área plantada e aumento significativo da capacidade instalada de moagem no Estado. De forma mais específica, busca-se estabelecer relações entre emprego/renda, saúde e escolaridade com a expansão da área plantada com cana-de-açúcar e com o aumento do 
número de unidades produtoras que moem essa matéria-prima para a produção de açúcar e etanol.

$\mathrm{Na}$ análise realizada,utiliza-se a metodologia de dados em painel, que tem algumas vantagens em relação às que usam dados de corte seccional ou séries temporais, o que será discutido adiante.

Este trabalho está dividido em cinco partes. Além desta breve introdução, na seção 2, apresenta-se uma revisão de literatura com os principais estudos relacionados à interação entre produção canavieira e desenvolvimento econômico. Na seção 3, descrevem-se a metodologia e as fontes dos dados utilizados; na seção 4, expõe-se e discutem-se os resultados encontrados, e, por fim, são apresentadas as principais conclusões deste estudo.

\section{2_Revisão de literatura}

\section{1_Medição de impactos socioeconômicos, bem-estar e desenvolvimento humano}

A análise de impactos socioeconômicos requer breve visitação à teoria do bem-estar. Mesmo havendo vasta literatura que trata disso, não existe consenso quando se busca definir as medidas de bem-estar, porque o assunto envolve aspectos normativos, possuindo imperfeições do ponto de vista teórico e dificuldades no campo prático. No campo teórico, as medidas de bem-estar são avaliadas por meio de funções de utilidade social; no campo prático, elas têm sido analisadas, na maior parte das vezes, por meio do conceito de renda real per capita, juntamente com o grau de desigualdade (Silva, 2008).

Uma das formas de medir o bem-estar é por meio da renda plena, expressa pela somatória da renda monetária, das transferências governamentais e dos subsídios (Grootaert, 1982). Do ponto de vista individual, a renda plena envolve um fluxo derivado de estoque de riqueza proveniente de riqueza física, associada a bens duráveis, riqueza financeira (ações, títulos do governo, etc.) e capital humano (riqueza relacionada às habilidades/treinos e ao talento natural). Esse fluxo divide-se em renda monetária - renda de salários, juros, transferências, subsídios - e renda não monetária satisfação no trabalho, satisfação no lazer, valor da produção própria, entre outras. Resume-se renda plena como um conjunto de oportunidades do indivíduo relacionadas ao consumo potencial e lazer.

O conceito de renda plena omite, no entanto, o impacto da incerteza sobre a perda de bem-estar individual e o fato de o bem-estar não depender apenas do consumo potencial, mas também de aspectos relacionados a fatores como saúde, segurança, educação e capacidades que incluem as dimensões, isto é, escolha e liberdade (Silva, 2008).

De maneira geral, a renda plena é de difícil mensuração, uma vez que requer dados que muitas vezes são indisponíveis. Assim, conclui-se ser impossível incluir em modelos que tratam de bem-estar dos indivíduos todos os fatores que o influenciam, dada a complexidade discutida.

Busca-se, no presente estudo, a exemplo do que vem sendo feito por diversas instituições nacionais e internacionais, apropriar-se do conceito de desenvolvimento humano para identificar as externalidades positivas relacionadas à expansão do setor sucroenergético. $O$ conceito de desenvolvimento humano tem como premissa a circunstância de que a qualidade de vida da população é afetada por dimensões sociais, políticas e culturais, além das econômicas. Neste estudo, serão utilizados indicadores agregados para a análise pretendida, que englobam três dimensões: educação, saúde e renda/emprego.

\section{2_Setor sucroenergético e desenvolvimento socioeconômico} Satolo e Bacchi (2012) realizaram estudo com o objetivo de avaliar os impactos da expansão do setor sucroenergético so- 
bre a renda da população nos municípios paulistas, sendo utilizado, para essa finalidade, um painel espacial dinâmico. A proporção de adultos na população municipal, a taxa de utilização da força de trabalho (total e apenas na agropecuária) e o rendimento médio dos vínculos empregatícios (total e apenas na agropecuária) foram utilizados como variáveis de controle.

Adicionalmente, para captar os impactos da expansão do setor sucroenergético sobre o PIB per capita (utilizado como proxy da renda per capita), a participação da agropecuária na área do município, a participação da agricultura na área da agropecuária, a participação da cana-de-açúcar na área da agricultura e uma dummy para os municípios com usina em operação foram incluídas no modelo. $\mathrm{O}$ estudo foi realizado com dados dos 645 municípios paulistas do período de 2000 a 2008. Os resultados do Método Generalizado de Momentos em Sistema - GMM-SYS - evidenciaram a existência de relação de dependência espacial e temporal no PIB real per capita dos municípios paulistas durante o período analisado. Os efeitos diretos e indiretos estimados indicam que a expansão do setor sucroenergético apresentou impacto positivo sobre o PIB per capita, tanto nos municípios onde a expansão ocorreu como também nos outros municípios mais próximos.

Anteriormente a esse estudo, outros buscaram avaliar a relação entre a expansão do setor sucroenergético e o crescimento econômico dos municípios. Silva (2008) avaliou os impactos do setor sucroenergético sobre o desenvolvimento socioeconômico dos municípios do Estado de São Paulo. A autora estimou modelos utilizando o Índice de Desenvolvimento Humano Municipal - IDH-M, de 2000, como proxy das condições socioeconômicas dos municípios paulistas. As variáveis para representar a presença de setor sucroenergético nos municípios foram: dummy, para indicar municípios com lavoura de cana-de-açúcar como atividade agrícola principal, e dummy, para representar municípios com usina operante. As variáveis de controle utilizadas foram: valor adicionado por setores de atividade econômica, rendimento médio dos vínculos empregatícios, média dos anos de estudo da população (15 a 64 anos de idade), grau de urbanização, densidade demográfica e também algumas que captam a prestação de serviços feita com recursos do Estado que afetam as condições de vida da população local.

A metodologia utilizada no aludido estudo foi a de Regressão Linear Múltipla e dois modelos foram empregados: de Efeitos Diretos e de Efeitos Cruzados (incluindo também séries formadas pela multiplicação das dummies com as demais variáveis explicativas). Depois de realizadas as modificações visando ao ajustamento do modelo, de forma a atender aos pressupostos do método (correção de heterocedasticidade e eliminação de variáveis linearmente relacionadas a outras), obteve-se resultado indicando que a presença do setor sucroenergético contribui positivamente para os IDHMs. No entanto, no caso do modelo de efeitos cruzados, isso não foi observado. Assim, conforme indicado pela própria autora, os resultados do estudo não foram conclusivos.

Walter et al. (2008) mostraram que, em 2000, os municípios com usinas ou com produção significativa de cana-de-açúcar ${ }^{1}$ apresentaram renda per capita estatisticamente maior do que a observada nos demais municípios. Também Spavorek et al. (2009), quantificando os efeitos da mudança no uso da terra, verificaram que os municípios com presença do setor sucroenergético apresentaram crescimento maior do PIB do que os demais. Contrariamente, Deuss (2012) não encontrou evidências da existência de uma relação causal entre o crescimento do setor sucroenergético e o da economia dos municípios paulistas entre 2002 e 2006.

Oliveira (2011) desenvolveu um estudo com o objetivo de comparar indicadores socioeconômicos em municípios dos Estados de São Paulo, Paraná, Minas Gerais, na região 
Centro-Sul, e de Alagoas e Pernambuco, na região Nordeste, escolhidos pela representatividade das atividades do setor sucroenergético. A autora utilizou nove indicadores e dois índices que expressam aspectos relacionados à educação, distribuição de renda, saúde/longevidade e desenvolvimento como um todo. Para São Paulo, um único índice (IPRSÍndice Paulista de Responsabilidade Social da Fundação Seade) foi analisado, considerando as décadas dos anos 2000, 2002, 2004 e 2006. Foram comparados, por meio de testes estatísticos, as médias e os desviospadrão de cada um dos indicadores entre os grupos de municípios com e sem atividades sucroalcooleiras. A comparação entre grupos foi feita considerando, entre outros: municípios com produção de cana e sem ela; com produção de cana e usinas instaladas; municípios que tradicionalmente produziram cana em todos os anos investigados. Os melhores resultados foram obtidos para os municípios que têm significativa produção de cana. Essa conclusão é clara para o Estado de São Paulo, e em menor grau para Alagoas, Paraná e Pernambuco. Em Minas Gerais, a vantagem do grupo de municípios canavieiros não é clara para a maioria dos indicadores analisados.

Em Chagas, Toneto e Azzoni (2010a), um modelo propensity score matching espacial foi utilizado para estimar o efeito da produção de cana-de-açúcar sobre o IDH-M. Os resultados indicaram que a presença do setor não é relevante para determinar as condições sociais nas localidades produtoras. Essa metodologia também foi usada por Chagas, Toneto e Azzoni (2010b),com o intuito de analisar o impacto da expansão do setor sucroenergético sobre a receita fiscal dos municípios paulistas. Já em Chagas, Toneto e Azzoni (2011), um modelo propensity score matching espacial foi empregado para avaliar o efeito da produção de cana-de-açúcar sobre o crescimento do PIB per capita, e, neste caso, os resultados indicaram que as regiões onde o cultivo da cana-de-açúcar foi expandido apresentaram crescimento do PIB per capita maior do que o observado em outras regiões onde a expansão da cana-de-açúcar poderia ter acontecido, mas não ocorreu.

\section{3_Metodologia}

Os modelos que tratam de agrupamento de dados permitem estabelecer relações econômicas explorando simultaneamente efeitos ao longo do tempo e entre diferentes unidades (indivíduos). As unidades podem representar um conjunto de países, regiões, setores, empresas, consumidores, etc. No presente estudo, as unidades referem-se aos municípios do Estado de São Paulo.

De acordo com Greene (2008), a técnica de agrupamento de dados de séries temporais e de corte seccional permite uma estimação mais eficiente dos modelos econométricos. O uso desses modelos tem vantagens do ponto de vista de estimação, podendo-se citar, entre elas: maior número de observações, o que permite assegurar as propriedades assimptóticas dos estimadores; aumento do número de graus de liberdade; redução da multicolinearidade, uma vez que os dados entre as unidades apresentam estruturas diferentes.

Um modelo de dados agregados (série temporal e corte seccional) pode ser representado por:

$$
Y_{i t}=a+b_{1} X_{i t 1}+b_{2} X_{i t 2}+\ldots+u_{i t}
$$

Com $i=1, \ldots N$ sendo as unidades e $t=1, \ldots T$ os períodos de tempo. $O$ número de observações é $N x T$.

Existem diferentes tipos de modelos de agrupamento de dados, a saber:

_Pooled, representado pela equação (1), na qual se assume que os parâmetros $a$ e $b_{i}$ são comuns para todas as unidades. Esse método pode ser adequado para amostras com unidades selecionadas a priori, que apresentam semelhanças 
em suas características estruturais. Esse modelo pode ser estimado pelo método dos Mínimos Quadrados Ordinários (MQO), admitindo-se que os erros são ruído branco e não se encontram correlacionados com os regressores, isto é, $\operatorname{Cov}\left(X_{i t}, u_{i t}\right)=0$.

De acordo com Stock e Watson (2007), a técnica de agrupamento de dados de séries temporais e de corte seccional permite o tratamento da heterogeneidade entre as unidades, quando existente. Dois modelos são propostos com essa finalidade:

_Efeitos fixos, em que uso desses modelos possibilita o controledos efeitos de variáveis omitidas (por indisponibilidade de dados e/ou por questões de graus de liberdade), que são diferentes entre as unidades. Nesse caso, a estimação é feita assumindo que a heterogeneidade das unidades é captada pela constante:

$$
Y_{i t}=a_{i}+b X_{i t}+\ldots+u_{i t}
$$

Sendo assim, $a_{i}$ capta, por exemplo, o efeito de dimensão de países/estados/municípios, de recursos naturais existentes, etc. $\mathrm{O}$ modelo de efeito fixo é a melhor alternativa para modelar dados em painel quando o intercepto é correlacionado com as variáveis explicativas. Também é desejável utilizar efeitos fixos quando as observações são obtidas de toda a população, e o que se deseja fazer são inferências para as unidades cujos dados estão disponíveis (Duarte; Lamounier; Takamatsu, 2012).

Existem três métodos para estimar os modelos em painel com efeitos fixos:

\section{1_O método de estimação com variáveis dummies}

Neste método, são introduzidas variáveis dummy para captar a heterogeneidade das unidades, distinguindo os seguintes casos particulares: (i) Efeitos Fixos Unilaterais Unidade: em que se utiliza uma dummy para cada unidade; (ii) Efeitos Fixos
Unilaterais Tempo: em que se utiliza uma dummy para cada período; (iii) Efeitos Fixos Bilaterais: modelo no qual se utilizam dummies para unidades e períodos.

\section{II_O método de estimação com primeiras diferenças}

Quando os efeitos fixos estão correlacionados com as variáveis explicativas, $\operatorname{Cov}\left(X_{i t} u_{i t}\right) \neq 0$, o método de Mínimos Quadrados Ordinários não produz estimadores consistentes. $\mathrm{O}$ problema resolve-se removendo os efeitos específicos individuais por meio das primeiras diferenças, podendo-se, então, utilizar o citado método.

\section{III_O método de estimação com as variáveis centradas}

O método consiste em centrar as variáveis, ou seja, subtrair de cada observação relativa às unidades a média temporal.

O modelo de painel com as variáveis centradas assume a seguinte forma:

$$
\left(Y_{i t}\right)_{T M}=b\left(X_{i t}\right)_{T M}+\ldots+v_{i t}
$$

em que $\left(Y_{i t}\right)_{T M}=\left(Y_{i t}-\bar{Y}_{i}\right)$ e $\left(X_{i t}\right)_{T M}=\left(X_{i t}-\bar{X}_{i}\right)$ são variáveis conhecidas como time-demeaned, com: $\bar{Y}_{i}=\frac{1}{T_{i}} \sum_{t=1}^{T_{i}} y_{i t} \mathrm{e}$ $\overline{X_{l}}=\frac{1}{T_{i}} \sum_{t=1}^{T_{i}} x_{i t}$, sendo as médias temporais das respectivas variáveis para cada unidade.

Efeitos aleatórios: $\mathrm{O}$ modelo com efeitos aleatórios assume a seguinte forma:

$$
\begin{aligned}
& Y_{i t}=\left(a+V_{i}\right)+b X_{i t}+\ldots+u_{i t} \\
& Y_{i t}=a+b X_{i t}+\ldots+\left(v_{i}+u_{i t}\right) \\
& Y_{i t}=a+b X_{i t}+\ldots+w_{i t}
\end{aligned}
$$

Podendo o termo de erro ser decomposto em: $w_{i t}=v_{i}+u_{i t}$ com: $v_{i} \sim N\left(0, \sigma_{v}^{2}\right)$

$$
u_{i} \sim N\left(0, \sigma_{u}^{2}\right)
$$


admitindo-se que todos os erros são homocedásticos e não autocorrelacionados.

A hipótese $\operatorname{Cov}\left(X_{i t}, u_{i t}\right)=0$, ou seja,de não haver correlação entre o erro $v_{i}$ (efeitos fixos não observados) e as variáveis explicativas, é crucial para que os estimadores sejam não viesados e consistentes. Todas as observações em cada unidade têm uma componente comum, o $v_{i}$, criando autocorrelação dos erros (within-unitautocorrelation), o que torna os estimadores de Mínimos Quadrados Ordinários não eficientes, e os erros padrão inválidos.

Segundo Hill, Griffiths e Judge (1999),nesse modelo o termo de erro possui média zero e variância constante. Os erros da mesma unidade em diferentes instantes do tempo são correlacionados; os erros de diferentes unidades no mesmo instante do tempo, não. Nesse caso, o método que fornece os melhores estimadores é o de Mínimos Quadrados Generalizados.

De forma geral, se as unidades sobre as quais se dispõe de dados são amostras aleatórias de uma população maior de unidades, o modelo de efeito aleatório é o mais indicado. Se existem razões para acreditar que os efeitos individuais estão correlacionados com as variáveis explicativas, o método de efeitos fixos é mais indicado (Cruz; Diaz; Luque, 2004). Os testes de Breusch-Pagan e de Hausman (Apêndice I) podem ser utilizados para definir a correta especificação do modelo de dados agrupados.

\section{1_Fonte e tratamento de dados}

Para a produção de cana-de-açúcar por município (em hectares), foram utilizadas as séries divulgadas pelo sistema Canasat do Instituto Nacional de Pesquisas Espaciais (INPE). Em relação à área municipal, foram empregados os dados do SIDRA/IBGE (transformado para hectare). $\mathrm{O}$ indicador de desenvolvimento utilizado foi o Índice FIRJAN de Desenvolvimento Municipal - IFDM -; além do índice conso- lidado, utilizaram-se também o indicador para três áreas, a saber: emprego/renda, educação e saúde. O indicador de desenvolvimento FIRJAN foi escolhido porque a sua periodicidade de divulgação e a disponibilidade de dados das outras variáveis do modelo permitiam que as séries tivessemo maior número de observações,comparativamente ao uso de indicadores alternativos.

Na Tabela 1,tem-se a composição do Índice FIRJAN de Desenvolvimento Municipal - IFDM -, na qual constam as variáveis utilizadaspara compor cada um dos indicadores: Emprego \& Renda, Educação e Saúde, utilizados para a construção do indicador agregado.

Tanto o valor adicionado pelo setor de serviços quanto o valor adicionado total são os divulgados pela Fundação Sistema Estadual de Análise de Dados (SEAD).Tais valores, expressos em termos nominais, foram transformados em valores reais utilizando o IGP-DI divulgado pela Fundação

Tabela 1_Indicadores utilizados no IFDM e seus respectivos componentes

\begin{tabular}{|c|c|c|}
\hline Emprego \& Renda & IFDM & Saúde \\
\hline Geração de emprego & Taxa de abandono & $\begin{array}{l}\text { Número de } \\
\text { consultas pré-natal }\end{array}$ \\
\hline $\begin{array}{l}\text { Estoque de } \\
\text { emprego formal }\end{array}$ & $\begin{array}{l}\text { Taxa de distorção } \\
\text { idade-série }\end{array}$ & $\begin{array}{l}\text { Óbitos infantis por } \\
\text { causas evitáveis }\end{array}$ \\
\hline \multirow[t]{3}{*}{$\begin{array}{l}\text { Salários médios do } \\
\text { emprego formal }\end{array}$} & $\begin{array}{l}\text { Percentual de } \\
\text { docentes com } \\
\text { ensino superior }\end{array}$ & $\begin{array}{l}\text { Óbitos por causa } \\
\text { não definidas }\end{array}$ \\
\hline & $\begin{array}{l}\text { Média de } \\
\text { horas aula diária }\end{array}$ & \\
\hline & $\begin{array}{l}\text { Resultado do Índice } \\
\text { de desenvolvimento } \\
\text { da educação básica } \\
\text { - IDEB . . . . . . . . . . }\end{array}$ & \\
\hline
\end{tabular}

Fonte: FIRJAN, 2012. 
Getúlio Vargas (FGV). Também foram obtidos na Fundação SEAD os valores de densidade populacional, cuja variável é medida em termos de habitantes $/ \mathrm{Km}^{2}$.

Ademais, construiu-se uma variável binária que indica a presença de usina ou destilaria de cana-de-açúcar no município. A variável assume valorı (um), caso o município possua, e 0 (zero), caso contrário.

As séries utilizadas foram transformadas em logaritmo para o ajustamento do modelo, exceto a variável binária que indica a presença de usina ou destilaria no município. Desta maneira, as relações entre as variáveis - valores dos coeficientes estimados - podem ser interpretadas como elasticidades. $\mathrm{O}$ estudo, realizado com dados anuais, compreende painéis de dados para o período de 2005 a 2009.

O pacote estatístico/econométrico utilizado foi o STATA 9.0.

\subsection{2_Modelo proposto para a análise do impacto da atividade sucroenergética no desenvolvimento socioeconômico}

Objetivando atender aos objetivos deste estudo, foram construídos quatro modelos teóricos, que possuem diferente variável dependente, como mostrado na Tabela 2, na qual constam também as correspondentes nomenclaturas.

Os modelos são expressos por:

$$
\text { IFDM }_{i t}=\left[D U ; \text { area } ; \text { densidade; } v a_{-} s\right]_{i t}^{\prime} \beta+z^{\prime}{ }_{i t} \alpha+\varepsilon_{i t}
$$

$I F D M \_E R_{i t}=\left[D U ; \text { area; densidade; } v a_{-} s\right]_{i t} \beta+z_{i t}^{\prime} \alpha+\varepsilon_{i t}$ (в)

$I F D M_{-} S_{i t}=\left[D U ; \text { area;densidade; } v a_{-} s\right]_{i t}{ }^{\prime} \beta+z^{\prime}{ }_{i t} \alpha+\varepsilon_{i t} \quad$ (C)

$I F D M_{ـ} S_{i t}=\left[D U ; \text { area;densidade; } v a_{-} s\right]_{i t}{ }_{i t} \beta+z^{\prime}{ }_{i t} \alpha+\varepsilon_{i t} \quad$ (D)

sendo:

$D U$ : Variável binária para a existência de usina ou destilaria de cana-de-açúcar;

area: representa a proporção da área cultivada com cana em relação à área do município;

densidade: é a densidade demográfica;
Tabela 2_Nomenclatura usada para as variáveis

\begin{tabular}{l|l} 
Nomenclatura & Descrição da variável \\
\hline IFDM & $\begin{array}{l}\text { Índice FIRJAN de Desenvolvimento } \\
\text { Municipal consolidado }\end{array}$ \\
\hline IFDM_ER & $\begin{array}{l}\text { Índice FIRJAN de Desenvolvimento Municipal: } \\
\text { área Emprego \& Renda }\end{array}$ \\
\hline IFDM_E & $\begin{array}{l}\text { Índice FIRJAN de Desenvolvimento Municipal: } \\
\text { área Educação }\end{array}$ \\
\hline IFDM_S & $\begin{array}{l}\text { Índice FIRJAN de Desenvolvimento Municipal: } \\
\text { área Saúde } \ldots \ldots \ldots \ldots\end{array} \ldots . . . \ldots \ldots \ldots$
\end{tabular}

Fonte: Elaborada pelos autores.

Nota: Consideram-se os logaritmos de tais variáveis.

$v a \_s$ : é o valor adicionado pelo setor serviços em relação ao valor adicionado total no município (ambos em milhões de reais de 2009), usado como uma proxy de estrutura com maior nível de urbanização.

$\mathrm{Na}$ especificação, foram incluídas ainda quatro variáveis dummy, que representam os anos 2006, 2007, 2008 e 2009. O uso da variável dummy permite captar efeitos específicos de cada ano sobre a variável dependente e possibilita, desta forma, que o intercepto varie ao longo do tempo.

\section{4_Resultados e discussão}

As estatísticas relativas a cada variável utilizada no estudo do impacto das atividades sucroalcooleiras no desenvolvimento dos municípios do Estado de São Paulo são apresentadas na Tabela 4 (Apêndice II), considerando a média, o desviopadrão, os valores máximo e mínimo da amostra como um todo (overvall), para unidades (between) e para anos (within). Observa-se grande variabilidade entre os valores quando se trata de comparar unidades.

Ajustou-se, com a finalidade de realizar os testes estatísticos para identificar a necessidade ou não de corrigir heterogeneidade não observada na amostra, o modelo pooled, cujos resultados estão na Tabela 5 (Apêndice II),mesmo con- 
siderando que esse modelo possivelmente não seria o ideal para a análise proposta. Em seguida, foram ajustados painéis considerando efeitos fixos e efeitos aleatórios, sendo os resultados deste último modelo apresentados na Tabela $7 \mathrm{do}$ Apêndice II. Os resultados do modelo considerando efeitos fixos, nas suas diversas versões, são apresentados na Tabela 3 deste tópico, sendo essa a especificação identificada nos testes preliminares como a melhor alternativa metodológica.

Os resultados do teste de BreuschandPagan apontam para a rejeição da hipótese nula, de não existência de he- terogeneidade não observada, para os quatros modelos ( $a$, $b, c$ e $d$ ). Os resultados do teste de Hausman, por sua vez, permitem concluir que, para todos os modelos propostos, o melhor método é o de efeitos fixos. Os resultados desses testes, bem como os resultados do ajustamento do modelo pooled e de efeitos aleatórios, estão no Apêndice II.

Os resultados do modelo de efeitos fixos(Tabela 3 ) mostram que a variável $D U$ é estatisticamente significativa nos modelos $a, b$ e $d$, sendo seu efeito mais intenso sobre o indicador de emprego/renda.Municípios com presença de usinas/

Tabela 3_Estimação do modelo efeitos fixos

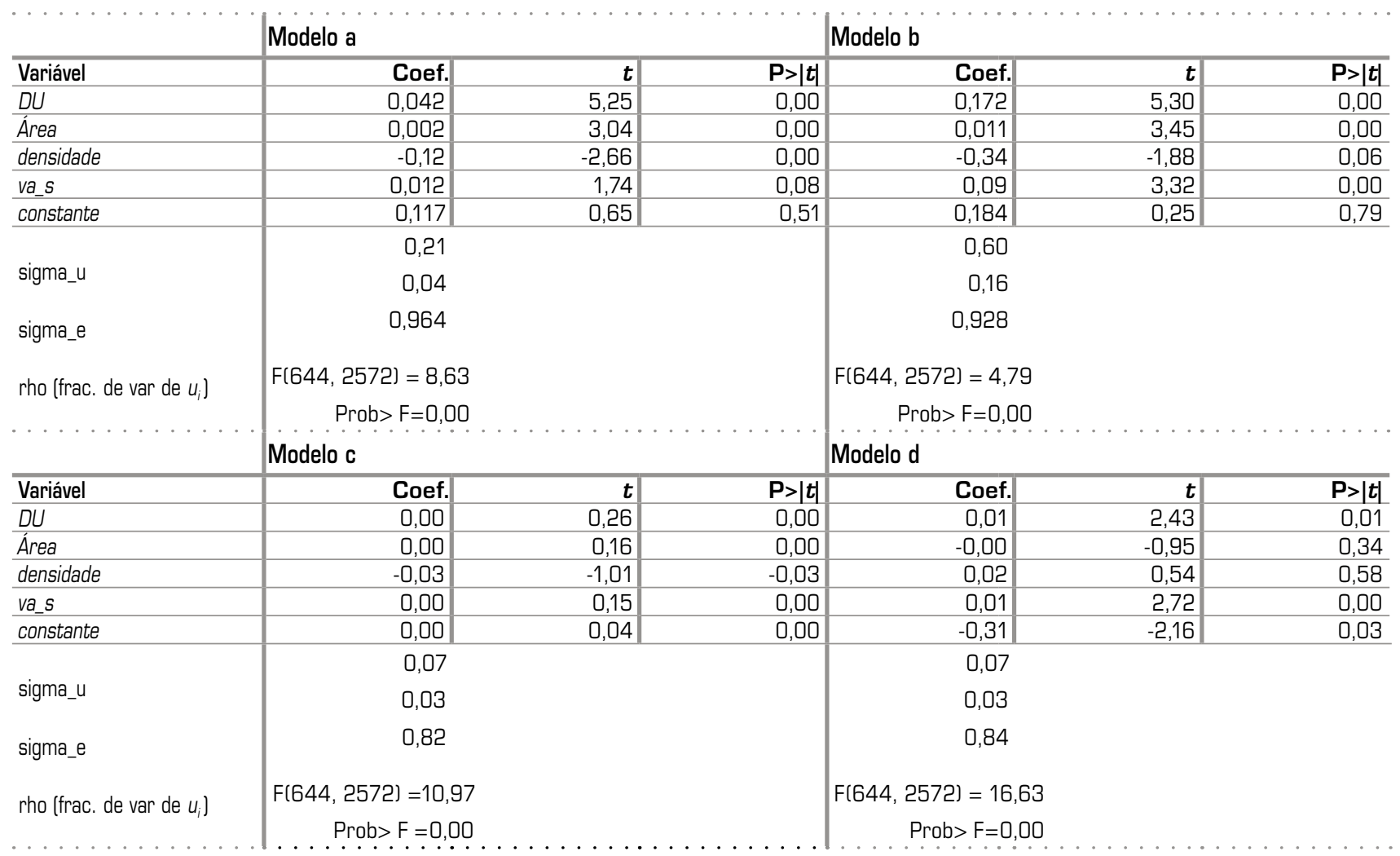

Fonte: Elaborada pelos autores com dados da pesquisa.

Nota: Os modelos apresentados possuem ainda quatro variáveis dummy para ano (D2006, D2007, D2008 e D2009), cujos coeficientes não foram apresentados. 
destilarias apresentam IFDM_ER 18,53\% maior que os que não possuem unidades produtoras. Embora significativos estatisticamente, o coeficiente da variável DU apresentou pequena magnitude tanto no caso do modelo em que a variável dependente era o indicador agregado quanto no caso em que tratava de aspectos de saúde. Esses resultados corroboram os encontrados por Satolo e Bacchi (2012) e Chagas, Toneto e Azzoni (2011), que mostram a importância do setor sucroenergético no que diz respeito ao aumento da renda municipal.

Por outro lado, os resultados relativos à educação e à saúde são compatíveis com os encontrados por Chagas, Toneto e Azzoni (2010a), que apontam a não existência de relação efetiva entre a expansão do setor sucroenergético e a melhoria desses fatores. Os resultados obtidos relacionados à educação e à saúde podem estar relacionados à uniformização dos benefícios oferecidos pelo Poder Executivo municipal ou estadual. Grande parte da população do Estado de São Paulo utiliza o Sistema Único de Saúde e frequenta escolas públicas na idade escolar.

Em relação ao coeficiente da variável area, os modelos $a$ e $b$ apresentam-se estatisticamente significativos, entretanto, as elasticidades são baixas. Para o indicador de emprego/ renda, a elasticidade é de aproximadamente 1,10\%; para o indicador consolidado, 0,20\%.Assim, pode-se observar que o efeito positivo da expansão do setor sucroenergético sobre emprego e renda está mais relacionado à presença de usinas/destilarias que à produção de cana.

\section{5_Conclusões}

Os resultados encontrados neste estudo permitem concluir que a expansão do setor sucroenergético no Estado de São Paulo tem efeitos positivos sobre o emprego e a renda, não se tendo observado evidências acerca de impactos positivos expressivos sobre a saúde e a educação.
O efeito da expansão do setor sobre emprego/renda ocorre em razão da existência de unidades que processam a matéria-prima - por exemplo, usina/destilaria de cana. Não se encontrou evidência de que a maior proporção de área com cana-de-açúcar tem impacto significativo sobre renda/emprego, saúde e educação. Mesmo sendo a atividade agrícola desse setor uma das que mais utilizam mãodeobra, os resultados obtidos permitem inferir que a agregação de valor às atividades agropecuárias é que traz benefícios econômicos consideráveis para a população da área de abrangência. Conclui-se, assim, que nos quesitos renda e emprego, a produção de açúcar, etanol e eletricidade impacta positivamente a população que reside nas proximidades das unidades produtoras.

Os resultados obtidos neste estudo permitem fazer inferências sobre a questão cana versus alimento. A tese de que há ameaça à segurança alimentar quando culturas importantes para a dieta da população brasileira são substituídas por cana-de-açúcar pode ser refutada. Primeiramente, cita-se o fato de que no Brasil há excedentes de produto de vários setores do agronegócio que acabam tendo como destino o mercado internacional, podendo-se citar a carne bovina, a carne de aves, os grãos necessários àprodução doméstica de proteína, etc. Menores excedentes poderiam ser gerados se houvesse a possibilidade de um consumo doméstico maior. O problema de segurança alimentar no Brasil, como discutido na literatura sobre o assunto, pode estar mais ligado à baixa renda do que à indisponibilidade de alimentos. Nesse sentido, a implantação de fábricas que agreguem valor à matéria-prima deve contribuir positivamente para a aquisição de alimentos, via emprego e renda maiores, mitigando problemas alimentares.

Outro aspecto que merece ser comentado quando se discutem aspectos relacionados à expansão do setor sucroenergético no país, embora não tenha sido o foco do presente 
estudo, diz respeito à substituição da colheita manual da cana por mecânica, que certamente reduz o impacto adverso da emissão de gases e fuligens. Em 2012/13, aproximadamente $85 \%$ da cana da região Centro-Sul foi colhida mecanicamente, diminuindo os problemas ambientais causados pela queima necessária na colheita manual. Em São Paulo, o percentual foi de $87 \%$.

Vale a pena também mencionar que, em função da tecnologia adotada nas diversas fases do processo produtivo do etanol, a United States Environmental Protection Agency (EPA)classificou o produto brasileiro como avançado, capaz de reduzir em pelo menos $50 \%$ as emissões de gazes que causam efeito estufa, comparativamente à gasolina. No caso do etanol brasileiro, esse percentual é ultrapassado por larga margem, podendo chegar a 90\%. O que pode, em parte, estar associado à baixa interação entre a atividade do setor sucroenergético e o indicador relacionado à saúde.

Acredita-se que a principal contribuição deste estudo foi a de analisar o efeito da expansão do setor sucroenergético do Estado de São Paulo sobre aspectos socioeconômicos considerados de forma individual, o que permitiu concluir que a população não teria benefícios diretos em relação à saúde e à educação, mas sim sobre renda/emprego.

\section{Nota}

${ }^{1}$ Os autores consideraram significativa a produção dos municípios que, em ordem decrescente, totalizava $90 \%$ da produção do Estado. 


\begin{abstract}
BACCHI, M.R.P. Interdependência dos mercados de gasolina Ce de álcool combustível no Estado de São Paulo.

2007. 150 p. Tese (Livre-Docência)

- Escola Superior de Agricultura

"Luiz de Queiroz", Universidade de São Paulo, Piracicaba, 2007.

BASE DE DADOS DO INSTUTO DE PESQUISAS ECONÔMICAS APLICADAS - IPEADATA. Dados Macroeconômicos. Disponível em: $<$ http://www.ipeadata.gov.br/ ipeaweb.dll/ipeadata?12236343>. Acesso em: 20 nov. 2012.
\end{abstract}

CHAGAS, A.L.S.; TONETO, R. Jr.; AZZONI, C.R. Avaliando os impactos da produção de canade-açúcar sobre indicadores sociais de regiões produtoras por meio de propensity score matching espacial. In: CONGRESSO DA SOCIEDADE BRASILEIRA DE ECONOMIA, ADMINISTRAÇ̃̃O E SOCIOLOGIA RURAL, 48., Campo Grande. Anais... SOBER, Brasília, 2010 a.1 CD-ROM.

CHAGAS, A.L.S.; TONETO, R. Jr.; AZZONI, C.R. Sugar cane, land use and regional development. Texto para Discussão 009/2011. Programa de Pós-Graduação em Economia Aplicada Universidade Federal de Juiz de Fora, Juiz de Fora, 2011.
CHAGAS, A.L.S.; TONETO, R. Jr.; AZZONI, C.R. The expansion of sugar canecultivation and its impacts on municipal revenues. In: AMANN, E.; BAER, W.; COES, D.V. (Eds.). Energy, biofuels and development: comparing Brazil and the United States. New York: Routledge, 2010b.

CRUZ, H.N.; DIAZ, M. D. M.; LUQUE, C. A. Metodologia de avaliação de custos nas universidades públicas: economias de escala e escopo. Revista Brasileira de Economia, Rio de Janeiro, v.58, n.1, p.45-66, 2004.

DEUSS, A. The economic growth impacts of sugarcane expansion in Brazil: an inter-regional analysis. Journal of Agriculture Economics, New York, v.63, n.3, p.528-551, Sept., 2012.

\section{DUARTE, C.P.; LAMOUNIER}

W.M.; TAKAMATSU, R.T.

Modelos econométricos para dados em painel: aspectos teóricos, exemplos de aplicação à pesquisa em contabilidade $\mathrm{e}$ Finanças. Rev. Adm., São Paulo, v.47, n.2, 2012.

\section{FEDERAÇÃO DAS INDÚSTRIAS} DO ESTADO DO RIO DE JANEIRO - FIRJAN. Índice FIRJAM de Desenvolvimento Municipal. Disponível em: $<$ http://www. firjan.org.br/ifdm/>. Acesso em: 20 nov. 2012.
FUNDAÇÃO GETÚLIO VARGAS - FGV. Disponível em: <http://www. agroanalysis.com.br/ index. php?area $=$ mercadonegocios $>$. Acesso em: 18 nov. 2012.

\section{FUNDAÇÃO SISTEMA} ESTADUAL DE ANÁLISE DE DADOS - SEAD. Índice Paulista de Responsabilidade Social. Disponível em: $<$ http://www. seade.gov.br/projetos/iprs/\#a $>$. Acesso em: 12 dez. 2012.

GREENE, W.H. Econometric Analysis. 6. ed. New Jersey: Prentice Hall, 2008. $1178 \mathrm{p}$.

GROOTAERT, C.The conceptual basis of measures of household welfare and their implied survey data requirements. WorkingPaper n. 19. Washington, DC: World Bank, 1982.

HILL, R. C.; GRIFFITHS, W. E.; JUDGE, G. G. Econometria. São Paulo: Saraiva, 1999.

INPE. MAPEAMENTO DE CANA VIA IMAGENS DE SATÉLITE DE OBSERVAÇÃO DA TERRA CANASAT. Tabelas de produção por município do Estado de São Paulo. Disponível em: $<$ http://www. dsr.inpe.br/laf/canasat/tabelas. html>. Acesso em: 10 out. 2012.
NASSAR, A.M.; RUDORFF, B.F.T.; ANTONIAZZI, L.B.; AGUIAR, D.A. DE; BACCHI, M.R.P., ADAMI, $M$. Prospects of the sugarcane expansion in Brazil: impacts on direct and indirect land use changes. In: ZUURBIER, P., VOOREN, J. van der. Contributions to climate change mitigation and the environment. Wageningen Academic Publishers,

Wageningen, 2008. p.63-93. Disponível em: $<$ http://www. mdpi.com/2071-1050/4/4/574>. Acesso em: 20 jun. 2012.

OLIVEIRA, J. G. de. Indicadores socioeconômicos em estados produtores de cana-de-açúcar: análise comparativa entre municípios. $2011.202 \mathrm{f}$. Tese (Doutorado em Planejamento de Sistemas Energéticos) Faculdade de Engenharia Mecânica, UNICAMP, Campinas, 2011.

SATOLO, L.F.; BACCHI, M.R. Impactos da recente expansão do setor sucroenergético sobre a renda per capita municipal no Estado de São Paulo. In: ENCONTRO BRASILEIRO DE ECONOMETRIA. 34., Porto Seguro. Anais... Porto Seguro: Sociedade Brasileira de Econometria, 2012. 
SILVA, R. Setor sucroenergético no Estado de São Paulo: mensurando impactos socioeconômicos. In: Workshop do Observatório do Setor sucroenergético, Ribeirão Preto, 2008.

\section{SISTEMA DO INSTITUTO}

BRASILEIRO DE GEOGRAFIA E ESTATÍSTICA DE RECUPERAÇÃO AUTOMÁTICA - SIDRA/

IBGE. Produção agrícola municipal. Disponível em: <http://www. sidra.ibge.gov.br/bda/acervo/ acervo2 $>$. Acesso em: 14 maio 2013.

SPAVOREK, G.; BARRETO, A.; BERNDES, G.; MARTINS, S., MAULE, R. Environmental, land-use and economic implications of Brazilian sugarcane expansion 1996-2006. Mitig Adapt Strateg Glob Change, p. 285-298, 2009.

STOCK, J.; WATSON, M.

Introduction to Econometrics. 2.ed. São Paulo: Pearson, 2007.

WALTER, A.; DOLZAN, P.; QUILODRÁN, O.; GARCIA, J. SILVA; C. DA, PIACENTE, F.; SEGERSTEDT, A. A sustainability analysis of the Brazilian ethanol In: Biocombustivel brasileiro: uma análise de sustentabilidade. Texto para discussão: UNICAMP, Campinas, 2008.

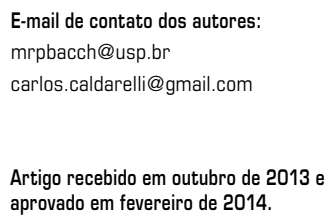




\section{Teste de Breusch-Pagan}

De acordo com Greene (2008), o teste de Breush-Pagan é utilizado para decidir qual dos modelos é o mais apropriado: o modelo pooled $\left(H_{0}\right)$ ou $\left(H_{A}\right)$ o modelo de efeitos aleatórios .

$$
\begin{array}{r}
H_{0}: \sigma_{v}^{2}=0 \quad \text { (constante comum - pooled, OLS) } \\
H_{A}: \sigma_{v}^{2} \neq 0 \quad \text { (efeitos aleatórios, GLS) }
\end{array}
$$

O teste de Breush-Pagan é um teste LM dado pela seguinte relação:

$$
L M=\frac{N T}{2(T-1)}\left[\frac{\sum_{i=1}^{N}\left(\sum_{t=1}^{T} \breve{w}_{i t}\right) \dagger}{\sum_{i=1}^{N} \sum_{t=1}^{T} w_{i t} \dagger}-1\right]^{2} \sim \chi_{1}^{2}
$$

\section{Teste de Hausman}

O teste de Hausman, descrito por Greene (2008), é utilizado para decidir qual dos modelos é o mais apropriado: o modelo de efeitos aleatórios $\left(\mathrm{H}_{0}\right)$ ou o modelo de efeitos fixos $\left(H_{A}\right)$. O teste apresenta-se da seguinte forma:

$$
\begin{array}{r}
H_{0}: \operatorname{Cov}\left(a_{i}, X_{i t}\right)=0 \quad \text { (efeitos aleatórios, GLS) } \\
\left.H_{A}: \operatorname{Cov}\left(a_{i}, X_{i t}\right) 7 \quad \text { (efeitos fixos, } L S D V\right)
\end{array}
$$

Sob a hipótese nula, os estimadores do modelo com efeitos aleatórios (estimação GLS) são consistentes e eficientes. Sob a hipótese alternativa, os estimadores GLS com efeitos aleatórios $(O L S)$ são não consistentes, mas os estimadores com efeitos fixos são.

A estatística de Hausman utilizada para testar essas hipóteses é a seguinte:

$$
H=\left(\breve{b}_{f e}-\breve{b}_{r e}\right)^{\prime}\left[\operatorname{Var}\left(\breve{b}_{f e}\right)-\operatorname{Var}\left(\breve{b}_{r e}\right)\right]^{-1}\left(\breve{b}_{f e}-\breve{b}_{r e}\right) \sim \chi_{k}^{2}
$$

Em que:

$\breve{b}_{f e}$ é o vetor dos estimadores do modelo com efeitos fixos

$\breve{b}_{r e}$ é o vetor dos estimadores do modelo com efeitos aleatórios

$\operatorname{Var}\left(\breve{b}_{f e}\right)$ é a matriz de variâncias-covariâncias dos estimadores $\breve{b}_{f e}$

$\operatorname{Var}\left(\breve{b}_{r e}\right)$ é a matriz de variâncias-covariâncias dos estimadores $\breve{b}_{r e}$, e $k$ é o número de regressores. 
Apêndice II

Tabela 4_Estatísticas das variáveis utilizadas

\begin{tabular}{|c|c|c|c|c|c|}
\hline Variável & Média & Desvio padrão & Mínimo & Máximo & Observações \\
\hline IFDM overvall & 0,75 & 0,067 & 0,54 & 0,95 & $\mathrm{~N}=3225$ \\
\hline between & & 0,061 & 0,60 & 0,92 & $n=645$ \\
\hline within & & 0,027 & 0,61 & 0,86 & $\mathrm{~T}=5$ \\
\hline IFDM_ER overvall & 0,53 & 0,16 & 0 & 1 & $\mathrm{~N}=3225$ \\
\hline between & & 0,15 & 0,28 & 0,95 & $n=645$ \\
\hline within & & 0,07 & 0,01 & 0,83 & $\mathrm{~T}=5$ \\
\hline IFDM_E overvall & 0,86 & 0,055 & 0,52 & 1 & $N=3225$ \\
\hline between & & 0,047 & 0,65 & 0,98 & $n=645$ \\
\hline within & & 0,029 & 0,68 & 1 & $\mathrm{~T}=5$ \\
\hline IFDM_S overvall & 0,85 & 0,059 & 0,55 & 1 & $N=3225$ \\
\hline between & & 0,054 & 0,65 & 0,97 & $n=645$ \\
\hline within & & 0,025 & 0,71 & 0,97 & $\mathrm{~T}=5$ \\
\hline areaovervall & 0,18 & 0,14 & 0 & 0,79 & $N=3225$ \\
\hline between & & 0,14 & 0 & 0,72 & $n=645$ \\
\hline within & & 0,12 & 0,01 & 0,74 & $\mathrm{~T}=5$ \\
\hline densidadeovervall & 291,49 & $1.158,76$ & 3,75 & $12.509,89$ & $N=3225$ \\
\hline between & & $1.159,31$ & 3,79 & $12.339,30$ & $n=645$ \\
\hline within & & 19,58 & 3,85 & 729,04 & $\mathrm{~T}=5$ \\
\hline va_sovervall & 0,60 & 0,13 & 0,090 & 0,93 & $N=3225$ \\
\hline between & & 0,12 & 0,11 & 0,92 & $n=645$ \\
\hline within & & 0,038 & 0,19 & 0,93 & $\mathrm{~T}=5$ \\
\hline
\end{tabular}

Fonte: Elaborada pelos autor

Tabela 5_Estimação por mínimos quadrados - regressão pooled

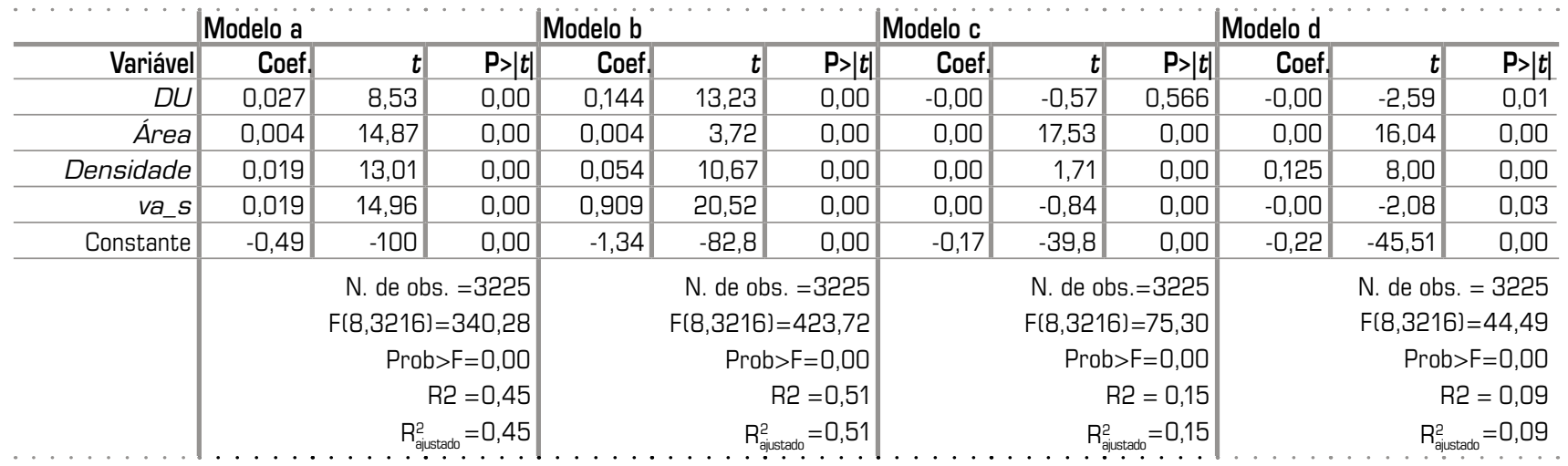

Fonte: Elaborada pelos autores com dados da pesquisa.

Nota: Os modelos apresentados possuem ainda quatro variáveis dummy para ano (D2006, D2007, D2008 e D2009), cujos coeficientes não foram apresentados. 
Tabela 6_Teste de Breuschand Pagan

\begin{tabular}{|c|c|c|}
\hline Especificação & $x^{2}(1)$ & Prob $>x^{2}$ \\
\hline Modelo a & 2331,25 & 0,00 \\
\hline Modelo b & 1185,86 & 0,00 \\
\hline Modelo c & 2796,76 & 0,00 \\
\hline Modelo d & 3598,39 & 0,00 \\
\hline
\end{tabular}

Fonte: Elaborada pelos autores com dados da pesquisa.

Tabela 7_Estimação do modelo efeitos aleatórios

\begin{tabular}{|c|c|c|c|c|c|c|c|c|c|c|c|c|}
\hline \multirow[b]{2}{*}{ Variável } & \multicolumn{3}{|l|}{ Modelo a } & \multicolumn{3}{|l|}{ Modelo b } & \multicolumn{3}{|l|}{ Modelo c } & \multicolumn{3}{|l|}{ Modelo d } \\
\hline & Coef. & $t$ & $P>|t|$ & Coef. & $t$ & $P>|t|$ & Coef. & $t$ & $P>|t|$ & Coef. & $t$ & $P>|t|$ \\
\hline$D U$ & 0,033 & 6,90 & 0,00 & 0,144 & 9,03 & 0,00 & 0,004 & 0,90 & 0,36 & 0,00 & 2,0 & 0,04 \\
\hline Área & 0,004 & 8,40 & 0,00 & 0,005 & 3,45 & 0,00 & 0,003 & 7,42 & 0,00 & 0,00 & 4,5 & 0,00 \\
\hline Densidade & 0,019 & 7,26 & 0,00 & 0,055 & 6,81 & 0,00 & $-0,00$ & $-0,10$ & 0,92 & 0,00 & 2,2 & 0,02 \\
\hline$v a_{-} s$ & 0,019 & 8,31 & 0,00 & 0,090 & 12,6 & 0,00 & 0,00 & 0,10 & 0,91 & 0,00 & 0,5 & 0,60 \\
\hline Constante & $-0,48$ & -63 & 0,00 & $-1,35$ & -57 & 0,00 & $-0,16$ & -22 & 0,00 & $-0,20$ & -25 & 0,00 \\
\hline sigma_u & 0,051 & & & 0,145 & & & 0,049 & & & 0,059 & & \\
\hline sigma_e & 0,041 & & & 0,167 & & & 0,035 & & & 0,033 & & \\
\hline rho (frac. de var) & 0,604 & & & 0,431 & & & 0,665 & & & 0,756 & & \\
\hline
\end{tabular}

Fonte: Elaborada pelos autores com dados da pesquisa.

Nota: Os modelos apresentados possuem ainda quatro variáveis dummy para ano (D2006, D2007, D2008 e D2009), cujos coeficientes não foram apresentados.

Tabela 8_Teste de Hausman

\begin{tabular}{l|r|r} 
Especificação & \multicolumn{1}{|l}{} & \\
\hline Modelo a & $x^{2}(8)=18,11$ & Prob $>x^{2}$ \\
\hline Modelo b & $x^{2}(8)=20,98$ & 0,02 \\
\hline Modelo c & $x^{2}(8)=39,81$ & 0,00 \\
\hline Modelo d & $x^{2}(8)=59,79$ & 0,00 \\
\hline & $\begin{array}{l}H_{0} \text { : diferença nos coeficientes } \\
\text { não sistemática }\end{array}$ & 0,00 \\
\hline
\end{tabular}

Fonte: Elaborada pelos autores com dados da pesquisa. 